Journal of Knot Theory and Its Ramifications

(C) World Scientific Publishing Company

\title{
ON THE REVERSIBILITY OF TWIST-SPUN KNOTS
}

\author{
CAMERON MCA. GORDON * \\ The University of Texas at Austin \\ Department of Mathematics, 1 University Station C1200, Austin, TX 78712-0257 \\ Received \\ Revised \\ ABSTRACT \\ Litherland has shown that if a knot is $(+)$-amphicheiral then its $m$-twist-spin is \\ reversible. We show that, for classical knots, in many cases the converse holds.
}

The irreversibility (sometimes called noninvertibility) of certain twist-spun knots has been established by Ruberman [10], using the Farber-Levine linking pairing and the Casson-Gordon invariants. More recently, alternative proofs of the irreversibility of the 2-twist-spun trefoil have been given by Carter, Jelsovsky, Kamada, Langford and Saito [4], and by Rourke and Sanderson [9], using quandle cohomology and the homotopy theory of racks, respectively. Similar methods have been used by Satoh [11] to prove the irreversibility of certain other twist-spun torus knots. In the present note we use more geometric methods to prove the following more general result.

Theorem 1. (1) The 2-twist-spin of a rational knot $\kappa$ is reversible if and only if $\kappa$ is amphicheiral.

(2) If $m, p, q$ are $>1$ then the $m$-twist-spin of the $(p, q)$ torus knot is irreversible.

(3) If $m \geq 3$ then the $m$-twist-spin of a hyperbolic knot $\kappa$ is reversible if and only if $\kappa$ is $(+)$-amphicheiral.

Since a rational knot is either hyperbolic or a $(2, q)$ torus knot, we obtain the following corollary.

Corollary 2. If $m \geq 2$ then the $m$-twist-spin of a rational knot $\kappa$ is reversible if and only if $\kappa$ is amphicheiral.

The "if" directions in parts (1) and (3) of Theorem 1 are due to Litherland [5], who shows that the $m$-twist-spin of a $(+)$-amphicheiral knot is always reversible.

We work in the PL category. A knot $\kappa$ (more precisely, an $n$-knot) is a locally flat oriented pair $\left(S^{n+2}, K\right)$, where $K$ is homeomorphic to $S^{n}$. (The knots in Theorem 1

*Partially supported by TARP grant 003658-0519-2001. 
are 1-knots.) Two knots $\kappa_{1}=\left(S^{n+2}, K_{1}\right)$ and $\kappa_{2}=\left(S^{n+2}, K_{2}\right)$ are equivalent if there is an orientation preserving homeomorphism of pairs $h:\left(S^{n+2}, K_{1}\right) \rightarrow$ $\left(S^{n+2}, K_{2}\right)$. A knot $\kappa=\left(S^{n+2}, K\right)$ is reversible if it is equivalent to $\left(S^{n+2},-K\right)$; it is $(\varepsilon)$-amphicheiral if it is equivalent to $\left(-S^{n+2}, \varepsilon K\right), \varepsilon= \pm$. Note that if $\kappa$ is reversible then $(+)$ - and $(-)$-amphicheirality coincide, and hence, since rational knots are reversible, we can unambiguously use the term amphicheiral in part (1) of Theorem 1 and in Corollary 2.

Let $\kappa=\left(S^{n+2}, K\right)$ be a knot. Then $K$ has a regular neighborhood $N(K)$, where $(N(K), K) \cong\left(S^{n} \times D^{2}, S^{n} \times\{(0,0)\}\right)[13]$, and the exterior of $\kappa$ is $X=$ $\overline{S^{n+2}-N(K)}$. Recall that $\kappa$ is fibered if $X$ is a fiber bundle over $S^{1}$; the fiber is then $M_{0}=\overline{M-B}$, where $M$ is a closed, connected, orientable $(n+1)$-manifold and $B$ is an $(n+1)$-ball in $M$, and $X$ is homeomorphic to the identification space $M_{0} \times I / f=M_{0} \times I /\left((x, 0) \sim(f(x), 1)\right.$ for all $\left.x \in M_{0}\right)$, for some orientation preserving homeomorphism $f: M_{0} \rightarrow M_{0}$, the monodromy of the bundle.

The observation that lies behind Theorem 1 is the following, the first part of which is due to Ruberman [10].

Proposition 3. Let $\kappa$ be a fibered knot with fiber $M_{0}$ and monodromy $f$. If $\kappa$ is reversible then $M_{0}$ and $-M_{0}$ are $h$-cobordant rel $\partial$. Moreover, the orientation reversing self-homotopy equivalence $g: M_{0} \rightarrow M_{0}$ induced by the $h$-cobordism satisfies $f g f \simeq g$.

Before giving the proof of Proposition 3, we show how it implies Theorem 1.

First note that attaching $B \times I$ to the $h$-cobordism between $M_{0}$ and $-M_{0}$ in the obvious way gives an $h$-cobordism between $M$ and $-M$. Also, the corresponding extension of $g$ to $M$ and any extension of $f$ to $M$ still satisfy $f g f \simeq g$.

Next recall Zeeman's theorem on twist-spinning [14]: if $\kappa$ is an $n$-knot, and $m$ is a positive integer, then $\kappa^{(m)}$, the $m$-twist-spin of $\kappa$, is an $(n+1)$-knot which is fibered with fiber $M_{0}$ and monodromy $f$, where $M$ is the $m$-fold branched cyclic covering of $\kappa$, and $f$ is the restriction to $M_{0}$ of the canonical covering translation of $M$.

\section{Proof of Theorem 1}

(1) If $\kappa_{p / q}$ is the rational knot associated with the rational number $p / q$, then the 2 -fold branched covering of $\kappa_{p / q}$ is the lens space $L(p, q)$. Hence Proposition 3 implies that if the 2-twist-spin $\kappa_{p / q}^{(2)}$ is reversible then $L(p, q)$ and $-L(p, q)$ are $h$ cobordant. By the $G$-signature theorem (see $[1, \mathrm{p} .479]$ ), two lens spaces are $h$ cobordant if and only if they are homeomorphic as oriented manifolds. Hence if $\kappa_{p / q}^{(2)}$ is reversible then $q^{2} \equiv-1(\bmod p)$, which implies that $\kappa_{p / q}$ is amphicheiral [12]. (Since $\kappa_{p / q}$ is reversible, $(+)$ - and (-)-amphicheirality coincide.) On the other hand, for any $n$-knot $\kappa$, if $\kappa$ is $(+)$-amphicheiral then $\kappa^{(m)}$ is reversible [5].

(2) Let $\tau_{p, q}$ denote the $(p, q)$ torus knot. The $m$-fold branched cyclic covering $M$ of $\tau_{p, q}$ is a Seifert fiber space. Since $\tau_{2, q}$ is a non-amphicheiral rational knot, we 
may assume by part (1) that either $m>2$ or $p$ and $q$ are both $>2$. Then $M$ is not a lens space (including $S^{3}$ and $S^{2} \times S^{1}$ ); see for example [8, Theorem 1]. Also, the Euler number $e(M) \neq 0$, by [7, Theorem 1.2] (see [8]). Hence by [7, Theorem 8.2], $M$ admits no orientation reversing self-homotopy equivalence. It follows from Proposition 3 that $\tau_{p, q}^{(m)}$ is irreversible.

(3) If $\kappa$ is the figure eight knot then $\kappa$ is $(+)$-amphicheiral, and hence $\kappa^{(m)}$ is reversible [5], so the theorem holds in this case.

If $\kappa$ is a hyperbolic knot other than the figure eight knot, and $m \geq 3$, then the $m$-fold branched cyclic covering $M$ of $\kappa$ is hyperbolic, and the canonical covering translation $f: M \rightarrow M$ is an isometry [2], [3]. Let $\tilde{K}$ be the (geodesic) fixed point set of $f$, and let $N$ be a tubular neighborhood of $\tilde{K}$, consisting of all points of $M$ within some sufficiently small distance of $\tilde{K}$. Note that $N$ can be parametrized as $S^{1} \times D$, where each meridian disk $\{x\} \times D$ is a geodesically embedded copy of the disk $D$ of some radius centered at the origin $(0,0)$ in the disk model of $\mathbb{H}^{2}$, and where $\tilde{K}=S^{1} \times\{(0,0)\}$. Then $f(N)=N$, and, taking polar co-ordinates on $D$, $f \mid N$ is given by $f(x,(r, \theta))=\left(x,\left(r, \theta+\frac{2 \pi}{m}\right)\right)$.

Now suppose that $\kappa^{(m)}$ is reversible, and let $g: M \rightarrow M$ be the degree -1 homotopy equivalence given by Proposition 3. By [6], $g \simeq \gamma$, where $\gamma$ is an isometry. Since $f \gamma f \simeq \gamma$, we have, again by [6], that $f \gamma f=\gamma$. In particular, $\gamma(\tilde{K})=\tilde{K}$. There are two possibilities: (i) $\gamma \mid \tilde{K}$ is orientation preserving, and (ii) $\gamma \mid \tilde{K}$ is orientation reversing.

In case (ii), $\gamma \mid N$ is of the form $\gamma(x, d)=\left(\alpha(x), \beta_{x}(d)\right)$, where $\beta_{x}: D \rightarrow D$ is some orientation preserving isometry. Hence $\beta_{x}$ is given by $\beta_{x}(r, \theta)=\left(r, \theta+\theta_{x}\right)$, for some $\theta_{x}$. Then $f \gamma f(x,(r, \theta))=\left(\alpha(x),\left(r, \theta+\theta_{x}+\frac{4 \pi}{m}\right)\right)$, and hence, since $m \geq 3$, $f \gamma f \neq \gamma$, a contradiction.

It follows that case (i) must hold. Since $f \gamma f=\gamma, \gamma$ induces an orientation reversing homeomorphism $h: S^{3} \rightarrow S^{3}$, such that $h(K)=K$ and $h \mid K$ is orientation preserving. Thus $\kappa$ is $(+)$-amphicheiral.

As noted above, the converse is proved in [5].

\section{Proof of Proposition 3}

The first part of the statement is due to Ruberman [10]; we include a proof for completeness. Let $X$ be the exterior of $\kappa$, so we have $X \simeq M_{0} \times I / f$. Suppose that $\kappa$ is reversible. Then we have an orientation preserving homeomorphism $h: S^{n+2} \rightarrow S^{n+2}$ such that $h(K)=K$ and $h \mid K$ is orientation reversing. By an isotopy we may assume that $h(N(K))=N(K)$, and that, under the homeomorphism $(N(K), K) \cong\left(S^{n} \times D^{2}, S^{n} \times\{(0,0)\}\right), h \mid N(K)=\alpha \times \beta$, where $\alpha: S^{n} \rightarrow S^{n}$ is some orientation reversing homeomorphism, and $\beta: D^{2} \rightarrow D^{2}$ is given by $\beta(r, \theta)=(r,-\theta)$. Lifting the restriction $h \mid X$ to the infinite cyclic covering of $X$, we get an orientation preserving homeomorphism $\tilde{h}: M_{0} \times \mathbb{R} \rightarrow M_{0} \times \mathbb{R}$, such that $\tilde{h} \mid S^{n} \times \mathbb{R}=\alpha \times \varepsilon$, where $\varepsilon: \mathbb{R} \rightarrow \mathbb{R}$ is given by $\varepsilon(t)=-t$.

Let $M_{0}^{\prime}=\tilde{h}\left(M_{0} \times\{0\}\right)$, and choose $t \in \mathbb{R}, t>0$, so that $M_{0} \times\{t\}$ is disjoint from 
$M_{0}^{\prime}$. Let $W$ be the compact submanifold of $M_{0} \times \mathbb{R}$ cobounded by $M_{0} \times\{t\}$ and $M_{0}^{\prime}$. Orient $M_{0}$ and $\mathbb{R}$, and thereby $M_{0} \times \mathbb{R}$, and orient $M_{0}^{\prime}$ so that the induced orientation on $\partial M_{0}^{\prime}=S^{n} \times\{0\}$ is the same as that induced by $M_{0} \times\{0\}$. Thus $W$ is an oriented cobordism rel $\partial$ between $M_{0} \times\{t\}$ and $M_{0}^{\prime}$. Note that the homeomorphism $\tilde{h} \mid M_{0} \times\{0\}: M_{0} \times\{0\} \rightarrow M_{0}^{\prime}$ is then orientation reversing, since $\tilde{h} \mid S^{n} \times\{0\}:$ $S^{n} \times\{0\} \rightarrow S^{n} \times\{0\}$ is the orientation reversing homeomorphism $\alpha$. Hence $W$ is an oriented cobordism rel $\partial$ between $M_{0}$ and $-M_{0}$.

Now $\overline{M_{0} \times \mathbb{R}-W}=U \amalg V$, where $U=M_{0} \times[t, \infty)$ and $V=\tilde{h}\left(M_{0} \times[0, \infty)\right)$. Hence there is a strong deformation retraction $M_{0} \times \mathbb{R} \rightarrow W$. Since the inclusions of $M_{0} \times\{t\}$ and $M_{0}^{\prime}$ into $M_{0} \times \mathbb{R}$ are homotopy equivalences, it follows that $W$ is an $h$-cobordism.

Let $i_{0}: M_{0} \rightarrow M_{0} \times \mathbb{R}$ be the inclusion map $i_{0}(x)=(x, 0)$, and let $p: M_{0} \times \mathbb{R} \rightarrow$ $M_{0}$ be projection onto the first factor. Then the orientation reversing self-homotopy equivalence $g: M_{0} \rightarrow M_{0}$ induced by the $h$-cobordism $W$ is given by $g=p \tilde{h} i_{0}$.

The group of covering translations of the infinite cyclic covering $M_{0} \times \mathbb{R}$ of $X$ is generated by $T: M_{0} \times \mathbb{R} \rightarrow M_{0} \times \mathbb{R}$, where $T(x, t)=(f(x), t+1)$. Note that $\tilde{h}$ is the lift of $h$ that takes $S^{n} \times\{0\}$ to $S^{n} \times\{0\}$, and $\tilde{h} T$ is the lift of $h$ that takes $S^{n} \times\{0\}$ to $S^{n} \times\{-1\}$. Hence $\tilde{h} T=T^{-1} \tilde{h}$, giving $T \tilde{h} T=\tilde{h}$. Let $S: M_{0} \times \mathbb{R} \rightarrow M_{0} \times \mathbb{R}$ be given by $S(x, t)=(x, t+1)$. Observe that $i_{0} f=T S^{-1} i_{0}$, and that $f p=p T$. Then $f g f=f p \tilde{h} i_{0} f=p T \tilde{h} T S^{-1} i_{0}=p \tilde{h} S^{-1} i_{0} \simeq p \tilde{h} i_{0}=g$, since $S \simeq i d$.

\section{References}

[1] M.F. Atiyah and R. Bott, A Lefschetz fixed point formula for elliptic complexes: II. Applications, Ann. of Math. 88 (1968), 451-491.

[2] M. Boileau and J. Porti, Geometrization of 3-orbifolds of cyclic type, Astérisque 272 (2001).

[3] D. Cooper, C. Hodgson and S. Kerckhoff, Three-dimensional Orbifolds and Cone Manifolds, Memoirs of the Mathematical Society of Japan 5 (2000).

[4] J.S. Carter, D. Jelsovsky, L. Langford, S. Kamada and M. Saito, Quandle cohomology and state-sum invariants of knotted curves and surfaces, arXiv math.GT/9903135.

[5] R.A. Litherland, Symmetries of twist-spun knots, Knot Theory and Manifolds, Proceedings, Vancouver 1983, ed. D. Rolfsen, Lecture Notes in Mathematics 1144, Springer, 1985, pp. 97-107.

[6] G. Mostow, Strong Rigidity of Locally Symmetric Spaces, Ann. of Math. Studies 78, Princeton University Press, 1973.

[7] W.D. Neumann and F. Raymond, Seifert manifolds, plumbing, $\mu$-invariant and orientation reversing maps, Algebraic and Geometric Topology, Proceedings, Santa Barbara 1977, ed. K.C. Millett, Lecture Notes in Mathematics 664, Springer, 1978, pp. 163-196.

[8] V. Nuñez and E. Ramírez-Losada, The trefoil knot is as universal as it can be, preprint.

[9] C. Rourke and B. Sanderson, There are two 2-twist-spun trefoils, preprint.

[10] D. Ruberman, Doubly slice knots and the Casson-Gordon invariants, Trans. Amer. Math. Soc. 279 (1983), 569-588.

[11] S. Satoh, On cocycle invariants of twist-spun $(2, n)$-torus knots, preprint.

[12] H. Schubert, Knoten mit zwei Brücken, Math. Z. 65 (1950), 133-170. 
[13] C.T.C. Wall, Locally flat PL submanifolds with codimension two, Proc. Camb. Phil. Soc. 63 (1967), 5-8.

[14] E.C. Zeeman, Twisting spun knots, Trans. Amer. Math. Soc. 115 (1965), 471-495. 